\title{
Association between haptoglobin genotype and coronary artery stenosis in patient with premature coronary artery disease.
}

\author{
Yunying Wang ${ }^{1}$, Wei Li ${ }^{1}$, Kexu Sui ${ }^{2}$, Qiyi Zhang ${ }^{1}$, Xiaoying Ma ${ }^{{ }^{*}}$ \\ ${ }^{1}$ Department of Medicine, Qingdao Municipal Hospital, Qingdao University Medical College, Qingdao, Shandong, PR \\ China \\ ${ }^{2}$ Department of Neurology, Qilu Hospital of Shandong University (Qingdao), Qingdao, Shandong, PR China
}

\begin{abstract}
Background: Accumulating evidence implicates Haptoglobin $(\mathrm{Hp})$ gene polymorphism as a potential risk predictor for Coronary Artery Disease (CAD). We explored the association of $\mathrm{Hp}$ polymorphism with premature CAD and its severity within 165 unrelated Chinese patients and 116 healthy controls using genotyping, biochemical parameters, and the Gensini scoring system. Levels of HP and IL-17 in plasma were determined by ELISA.

Results: The frequencies of $\mathrm{Hp}$ genotypes and Alleles, Hp levels and IL-17 levels were significantly different between CVD group and control group, and among the three subgroups. Hp2-2 was associated with the highest Gensini score but the lowest $\mathrm{Hp}$ concentrations. After adjustments for confounding parameters, the OR of premature CAD associated with $\mathrm{Hp2}-2$ was 0.630 (95\% CI $0.449-0.884 ; \mathrm{P}<0.05)$. The Hp2-2 genotype was associated with an increase in coronary artery stenosis especially in group $\mathrm{C}$ $(O R=2.319 ; 95 \%$ CI 1.278-4.209; $\mathbf{P}<0.05)$. Gensini score was associated with Hp and IL-17 levels when we considered the entire patients. $r$-values were 0.296 and 0.140 , respectively $(P<0.05)$.

Conclusions: Hp polymorphism is independently associated with increasing CAD severity. Hp and IL-17 may be biomarker of coronary artery stenosis. Further studies are warranted to study the role of $\mathrm{Hp}$ and IL-17 in the pathogenesis of CAD.
\end{abstract}

Keywords: Haptoglobin, Genotype, Premature coronary artery disease, IL-17.

Accepted on February 15, 2018

\section{Introduction}

Atherosclerosis, the formation of lipid and cell-rich lesions on the intimal or lumenal surfaces of arteries leading to occlusion, thrombosis and infarction, is the primary determinant of the majority of cardiovascular disease mortality. Oxidative stress and inflammation play important roles in all phases of atheroma development. Haptoglobin $(\mathrm{Hp})$ is an acute phase protein that acts as an antioxidant by virtue of its ability to bind free hemoglobin $(\mathrm{Hb})$ and prevent heme-iron mediated oxidation, thereby inhibiting hemoglobin-induced oxidative damage. Hp contains two types of polypeptide chains: $\alpha$ and $\beta$ chain, which is common to the three phenotypes. The $\mathrm{Hp}$ gene contains two structural alleles: $\mathrm{Hp} 1$ and Hp2. Hp2 is the product of a non-homologous intragenic duplication of exons 3 and 4 of $\mathrm{Hp}$ and thus could be considered to be a copy number variant. The three major phenotypes of $H p$ are: an $\alpha 1-\beta$ dimer for Hp1-1; multiple $\alpha 2-\beta$ units for Hp2-2; and a combination of $\alpha 1-\beta$ dimer with multiple $\alpha 2-\beta$ units for $\mathrm{Hp} 2-1$. The Hp protein products of the Hp 1 and Hp 2 alleles differ in both their biochemical and their functional properties such as the ability to clear $\mathrm{Hb}$ from the plasmatic compartment. $\mathrm{Hp} 1-1$ is a better antioxidant and binds more strongly with free $\mathrm{Hb}$ than $\mathrm{Hp} 2-2$.
However, Hp2-2 is believed to have lower anti-inflammatory capacity. As $H p$ polymorphism has been associated with the prevalence and clinical evolution of many inflammatory diseases, including infections, atherosclerosis and autoimmune disorders, and $\mathrm{Hp}$ seems to have a protective role in reducing the severity of Th1/17-mediated inflammatory processes, we investigated the association between the haptoglobin genotype and IL-17 and the incidence of premature Coronary Artery Disease (CAD).

\section{Materials and Methods}

\section{Participants}

Experiments and procedures were approved by the Institutional Research Human Ethics Committee, Qingdao Municipal Hospital. From this hospital a total of 281 unrelated Chinese Han subjects, including 165 patients with premature coronary heart disease (CAD) and 116 healthy controls, were recruited. We assessed, using a questionnaire, family medical history (diabetes, hypertension and cardiovascular disease), personal medical history, drug intake and lifestyle. The 116 agematched and sex-matched control subjects were randomly 
selected from individuals participating in the same hospital for routine health examinations. They were judged to be free of hypertension and cardiovascular disease according to the history, clinical examination, electrocardiogram and treadmill test. All patients with coronary heart disease underwent coronary angiography and have $\mathrm{CAD}$, which was defined as $\geq$ $50 \%$ diameter narrowing in 1 major coronary artery or its major branches. Premature $\mathrm{CAD}$ was coded present for subjects with age $\leq 55$ in male and $\leq 65$ in female. The Gensini scoring system was used to calculate the severity of CAD $[1,2]$. It is a scoring system for evaluating collateral circulation of the coronary arteries and it assesses the extent of blockage. This method defines narrowing of the lumen of the coronary arteries as 1 for $25 \%-49 \%$ stenosis, 2 for $50 \%-74 \%$ stenosis, 4 for $75 \%-90 \%$ stenosis, 8 for $90 \%-98 \%$ stenosis, 16 for $99 \%$, and 32 for total occlusion. The score is then multiplied by a factor that represents the importance of the lesion's location in the coronary artery system. For the location scores, 5 points were given for the LMA; 2.5 for the proximal LAD or LCX; 1.5 for the mid segment LAD and LCX; 1 for the distal segment of LAD and LCX, first diagonal branch, first OM, RCA, PDA, and intermediate artery; and 0.5 for the second diagonal and second OM. The severity of angiographic CAD was generated as the sum of the above scores in all segments. We classified subjects with premature CAD into three subgroups according to Gensini score status as follows: (1) group A with Gensini score $<50$; (2) group B with Gensini score $\geq 50$ and $\leq 90 \mathrm{p}$; (3) group C with Gensini score $\geq 90$.

\section{Laboratory analysis}

Venous blood was drawn before coronary angioplasty and centrifuged, and then serum was immediately frozen in a refrigerator at $-80^{\circ} \mathrm{C}$. The levels of blood glucose, highsensitivity C-reactive protein (hs-CRP), total cholesterol, triglycerides, HDL-cholesterol and LDL-cholesterol were determined using routine methods at the Department of Laboratory Medicine, Qingdao Municipal Hospital. Levels of Hp and IL-17 in plasma were determined by ELISA.

\section{Measurement of Hp genotyping}

The Hp genotype was determined by PCR as described previously [3] with slight modifications. In brief, $25 \mu \mathrm{L}$ reactions contained $20 \mathrm{ng}$ genomic DNA, 2.5 units Taq Polymerase, 10X Tap plus Buffer (with $\mathrm{Mgcl}_{2}$ ) $2.5 \mu \mathrm{l}, 2.5$ mmol/LdNTP mixture $2 \mu \mathrm{l}$ and $0.5 \mathrm{nM}$ of each primer (A/B or $\mathrm{C} / \mathrm{D})$. Oligonucleotide primers $\mathrm{A}$ and $\mathrm{B}$ were used for amplification of a $1757 \mathrm{bp} \mathrm{Hp} 1$ allele-specific sequence. Primers C and D were used to amplify a 349 bp Hp 2 allele specific sequence. The primers A (A: 5'GAGGGGAGCTTGCCTTTCCATTG-3'), B (B: 5'GAGATTTTTGAGCCCTGGCTGGT-3'), C (C: 5'CCTGCCTCGTATTAACTGCACCAT-3') and D (D: 5'CCGAGTGCTCCACATAGCCATGT-3') were synthesized by Shanghai DNA Bio Technologies Co., Ltd. (Shanghai, China). Amplification was performed with an initial denaturation at $95^{\circ} \mathrm{C}$ for $2 \mathrm{~min}$, followed by 35 cycles of reaction at $95^{\circ} \mathrm{C}$ for
$60 \mathrm{~s}, 69^{\circ} \mathrm{C}$ for $30 \mathrm{~s}$, and $72^{\circ} \mathrm{C}$ for $60 \mathrm{~s}$, with a final extension at $72^{\circ} \mathrm{C}$ for $7 \mathrm{~min}$. The PCR products were separated by $1 \%$ agarose gel electropheresis, based on the length of the amplified fragments (Figure 1).

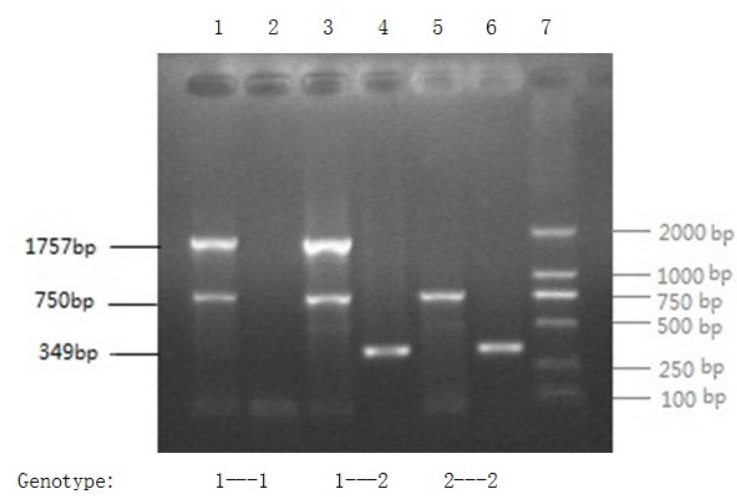

Figure 1. PCR products on 1\% agarose gel. Lanes 1 and 2: Hp1-1 genotype; lanes 3 and 4: Hpl-2 genotype; lanes 5 and 6: Hp2-2 genotype. Lane 7: marker.

\section{Statistical analysis}

Statistical analyses were performed using a commercially available statistical software package (SPSS17.0). Data were expressed as mean \pm Standard Deviation (SD). Variables were compared between patients and controls using Student's unpaired t-test for continuous variables and $\chi^{2}$ tests for categorical variables. The continuous variables of three subgroups were assessed by one-way Analysis of Variance (ANOVA). Significance was set at $\mathrm{p}<0.05$.

\section{Results}

\section{Characteristics of the participants}

The characteristics of subjects in the current study are detailed in Table 1. Mean age, male sex percentage and current smoking percentage of subjects with premature CAD was slightly higher than subjects in the control group, but there were no significant differences in patients versus controls. CVD group had significantly higher LDL levels, C-Reactive Protein (CRP) levels, LP $(\alpha)$ levels, and Hp levels than the control group did. IL-17 levels were also significantly different between CVD group and control group. In subgroups of patients, CRP levels and Hp levels showed significant differences, while IL-17 levels was not expressed at significant difference among three subgroups (Table 2). It was just slightly higher in groups $\mathrm{C}$ and $\mathrm{B}$ than group $\mathrm{A}$.

Table 1. Characteristics of the study participants.

\begin{tabular}{llll}
\hline & Patients & Controls & $\mathbf{P}^{*}$ \\
\hline $\mathrm{N}$ & 165 & 116 & \\
\hline Age & $51.9 \pm 3.8$ & $51.6 \pm 3.6$ & $>0.05$ \\
\hline Male sex N (\%) & $133(80.6)$ & $85(73.3)$ & $>0.05$ \\
\hline Current smoking (\%) & 36.2 & 33.9 & $>0.05$ \\
\hline
\end{tabular}



disease

\begin{tabular}{llll}
\hline Blood glucose $(\mathrm{mmol} / \mathrm{l})$ & $5.07 \pm 0.85$ & $4.93 \pm 0.68$ & $>0.05$ \\
\hline Total cholesterol $(\mathrm{mmol} / \mathrm{l})$ & $5.06 \pm 1.04$ & $4.87 \pm 0.95$ & $>0.05$ \\
\hline Triglyceride $(\mathrm{mmol} / \mathrm{l})$ & $1.92 \pm 0.58$ & $1.79 \pm 0.67$ & $>0.05$ \\
\hline $\mathrm{HDL}(\mathrm{mmol} / \mathrm{l})$ & $1.19 \pm 0.66$ & $1.32 \pm 0.58$ & $>0.05$ \\
\hline $\mathrm{LDL}(\mathrm{mmol} / \mathrm{l})$ & $2.87 \pm 0.76$ & $2.54 \pm 0.73$ & $<0.05$ \\
\hline $\mathrm{CRP}(\mathrm{mg} / \mathrm{l})$ & $15.13 \pm 3.92$ & $2.49 \pm 1.04$ & $<0.05$ \\
\hline $\mathrm{LP}(\mathrm{a})(\mathrm{mg} / \mathrm{dl})$ & $25.7 \pm 9.69$ & $19.2 \pm 8.74$ & $<0.05$ \\
\hline $\mathrm{Hp}(\mathrm{mg} / \mathrm{dl})$ & $136.3 \pm 19.7$ & $84.1 \pm 14.9$ & $<0.05$ \\
\hline $\mathrm{IL}-17(\mathrm{pg} / \mathrm{ml})$ & $91.8 \pm 18.6$ & $42.6 \pm 9.5$ & $<0.05$ \\
\hline $\mathrm{D}-\mathrm{dimers}(\mathrm{ng} / \mathrm{ml})$ & $422 \pm 70.3$ & $366 \pm 69.4$ & $<0.05$ \\
\hline
\end{tabular}

${ }^{\text {*}} \mathrm{P}$ values are for tests of any difference between patients and controls.

Table 2. Characteristics of the subgroups of patients.

\begin{tabular}{lllll}
\hline & \multicolumn{2}{l}{ Subgroups } & & P \\
\cline { 2 - 4 } & Group A & Group B & Group C & \\
\cline { 1 - 4 } $\mathrm{N}$ & 89 & 43 & 33 & $>0.05$ \\
\hline Male sex N (\%) & $77(86.5)$ & $33(76.7)$ & $23(69.7)$ & $>0.05$ \\
\hline CRP $(\mathrm{mg} / \mathrm{l})$ & $11.85 \pm 2.44$ & $15.02 \pm 3.82$ & $18.51 \pm 4.06$ & $<0.05$ \\
\hline Hp $(\mathrm{mg} / \mathrm{dl})$ & $18.51 \pm 4.06$ & $139.1 \pm 19.6$ & $151.2 \pm 21.5$ & $<0.05$ \\
\hline IL-17 $(\mathrm{pg} / \mathrm{ml})$ & $89.7 \pm 17.9$ & $91.5 \pm 18.3$ & $94.2 \pm 20.7$ & $>0.05$ \\
\hline D-dimers $(\mathrm{ng} / \mathrm{ml})$ & $421 \pm 60.2$ & $442 \pm 65.8$ & $454 \pm 69.6$ & $<0.05$ \\
\hline
\end{tabular}

\section{Genotypic frequencies of haptoglobin}

The genotypic frequencies of the Hp are shown in Table 3. These data in both groups were consistent with the distribution predicted by the Hardy-Weinberg equilibrium. In control subjects, the distribution of the Hp polymorphism at baseline

was $31.9 \%(\mathrm{n}=37)$ for the Hp1-1 type, $46.6 \%(\mathrm{n}=54)$ for the Hp 2-1 type, and 23.3\% ( $\mathrm{n}=27)$ for the Hp 2-2 type. Of the 165 CAD patients, these prevalence's were respectively, $18.8 \%$ $(\mathrm{n}=31), 52.7 \% \quad(\mathrm{n}=87)$ and $28.5 \%(\mathrm{n}=47)$, which showed significant difference compared with control group (Table 3). The individuals with the $\mathrm{Hp} 2$ allele were found to be more likely to have CVD (OR (95\% CI), 0.630 (0.449, 0.884), $\mathrm{P}<0.05$ ) (Table 4). In three subgroups of patients, $\mathrm{Hp}$ genotypes and allele frequencies emerged significant differences. Comparisons between any two subgroups showed that there were significant differences in genotypes and allele frequencies, with the exception of allele frequencies between Groups A and B (Table 4). Individuals with the Hp2 allele were found to be associated with a greater severity of coronary atherosclerosis.

Table 3. Frequencies of Hp genotypes and characteristics of the study participants according to Hp genotype.

\begin{tabular}{llllll}
\hline & Total & \multicolumn{2}{l}{ Haptoglobin genotype } & p \\
\cline { 3 - 5 } & & $1-1$ & $1-2$ & $2-2$ & \\
\cline { 3 - 5 } Patients n (\%) & 165 & $31(18.8)$ & $87(52.7)$ & $47(28.5)$ & \\
\hline Controls n (\%) & 116 & $39(33.6)$ & $53(45.7)$ & $24(20.7)$ & $<0.05^{*}$ \\
\hline Group A n (\%) & 89 & $20(22.5)$ & $53(59.5)$ & $16(18.0)$ & \\
\hline Group B n (\%) & 43 & $6(14.0)$ & $23(53.5)$ & $14(32.5)$ & \\
\hline Group C n (\%) & 33 & $5(15.2)$ & $11(33.3)$ & $17(51.5)$ & $<0.05^{\#}$ \\
\hline Hp (mg/dl) & & $154.6 \pm 39.5$ & $141.8 \pm 31.2$ & $88.2 \pm 28.7$ & $<0.05$ \\
\hline Gensini score & $22.5 \pm 13.8$ & $47.1 \pm 21.5$ & $65.5 \pm 31.6$ & $<0.05$ \\
\hline IL-17 (pg/ml) & $89.4 \pm 22.1$ & $91.6 \pm 11.8$ & $94.2 \pm 23.7$ & $>0.05$ \\
\hline
\end{tabular}

${ }^{*} \mathrm{P}$ values are for tests of $\mathrm{Hp}$ genotype difference between patients and controls. \#P values are for tests of $\mathrm{Hp}$ genotype difference between the 3 subgroups.

Table 4. Frequencies of Hp alleles in the study subjects.

\begin{tabular}{|c|c|c|c|c|c|c|c|}
\hline & \multirow[t]{2}{*}{$\mathrm{n}$} & \multicolumn{3}{|l|}{ Alleles } & \multirow[t]{2}{*}{ OR } & \multirow[t]{2}{*}{$95 \% \mathrm{Cl}$} & \multirow[t]{2}{*}{ p } \\
\hline & & 1 & 2 & p & & & \\
\hline Controls & 116 & $131(56.5)$ & $101(43.5)$ & & & & \\
\hline Patients & 165 & $149(45.2)$ & $181(54.8)$ & $<0.05^{*}$ & 0.63 & $0.449-0.884$ & $<0.05$ \\
\hline Group A & 89 & $93(52.2)$ & $85(47.8)$ & & Referent & & \\
\hline Group B & 43 & $35(40.7)$ & $51(59.3)$ & & 1.577 & $0.954-1.710$ & $>0.05$ \\
\hline Group C & 33 & $21(31.8)$ & $45(68.2)$ & $<0.05^{\#}$ & 2.319 & $1.278-4.209$ & $<0.05$ \\
\hline
\end{tabular}

*P values are for tests of $\mathrm{Hp}$ Alleles difference between patients and controls. $\mathrm{\#}$ values are for tests of $\mathrm{Hp}$ Alleles difference between the three subgroups.

\section{Association of Hp genotypes with biochemical parameters}

The Hp Genotype was significantly associated with the Hp concentration. The Hp levels were significant differences among three genotypes and remained significant after adjustment for HDL and LDL cholesterol, and in accordance with literature reports, showing highest values in the $\mathrm{Hp} \mathrm{1-1}$ group and the lowest in the Hp 2-2 individuals (Figure 2). Gensini score was significantly higher in the $\mathrm{Hp} \mathrm{2-2}$ individuals $(65.5 \pm 31.6)$ than in Hp 2-1 (47.1 \pm 21.5$)$ and Hp $1-1$ (22.5 \pm 13.8$)$ individuals, and there was a highly 
significant difference between any two genotypes (Table 3), and remained significant after adjustment for HDL and LDL cholesterol. CRP was also observed significant differences among three genotypes $(\mathrm{F}=10.153, \mathrm{P}<0.05)$. But there was no significant difference in IL17 by Hp genotypes (Hp 1-1: $89.4 \pm$ 22.1; Hp 1-2: $91.6 \pm$ 11.8; Hp 2-1: $94.2 \pm 23.7$; F=2.287; $\mathrm{P}>0.05)$. Comparisons of IL17 between any two genotypes showed no significant differences in Hp 1-1 and Hp 1-2 and in Hp 1-2 and $\mathrm{Hp} 2-2$, with the exception between $\mathrm{Hp} 1-1$ and $\mathrm{Hp}$ $2-2(q=10.231, \mathrm{P}<0.05)$. Associations of Hp and IL-17 levels with Gensini score were judged by Spearman correlation coefficients. We observed a significant positive correlation between Gensini score and Hp levels (Figure 3), and between Gensini score and IL-17 levels (Figure 4) when we considered the entire patients. r-values were 0.296 and 0.140 , respectively, $\mathrm{P}<0.05$.

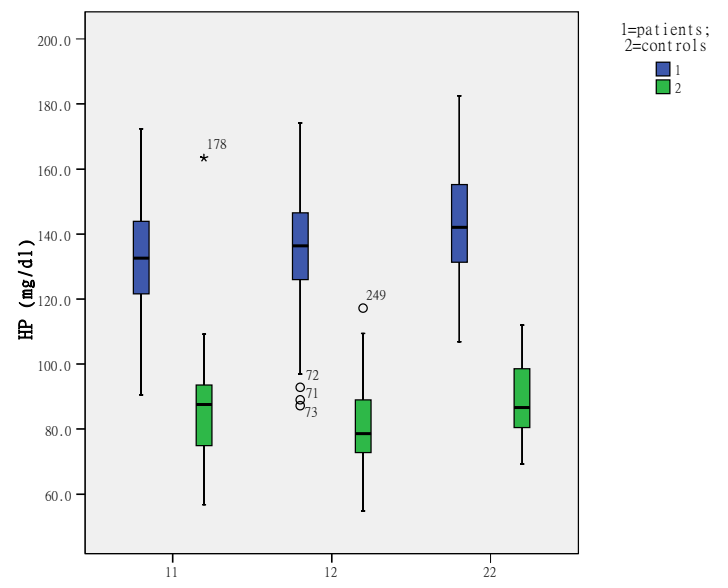

Figure 2. The distribution of Hp concentration among three HP genotypes.

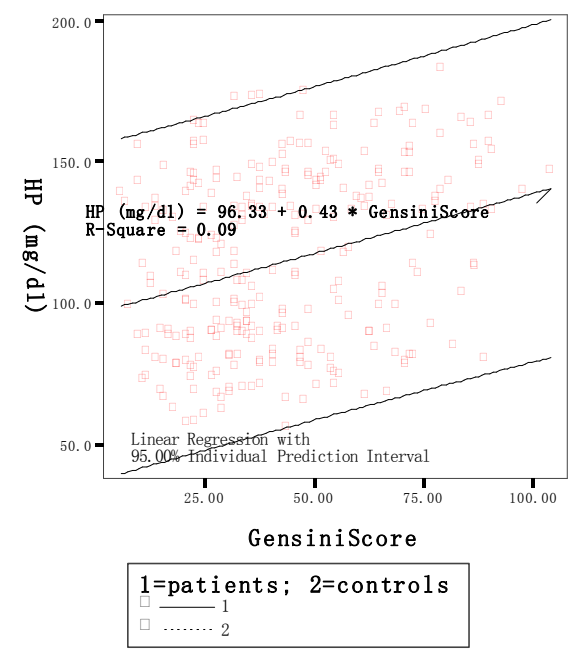

Figure 3. Relationship between HP levels and Gensini score.

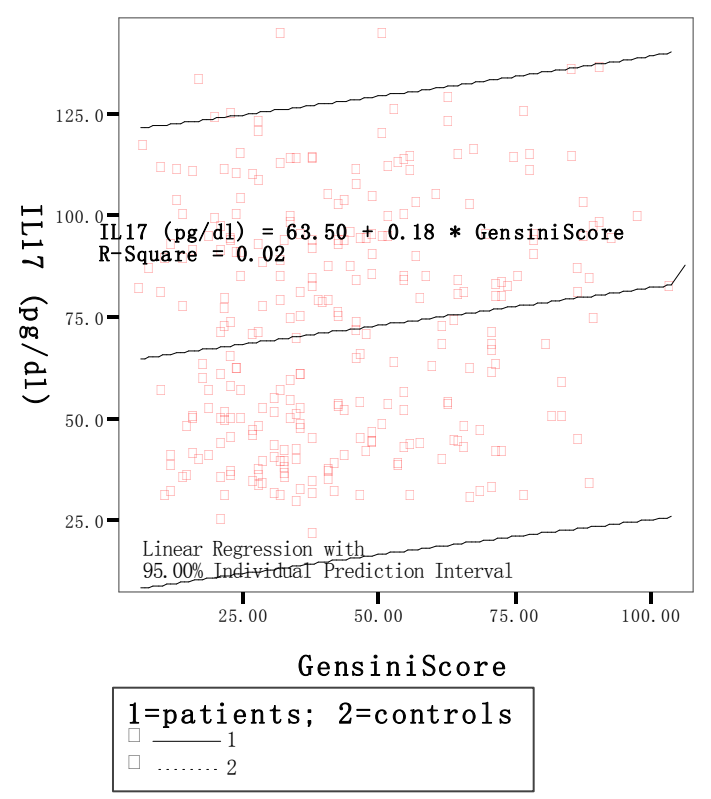

Figure 4. Relationship between IL-17 levels and Gensini score.

\section{Discussion}

In the present study, we investigated the association of $\mathrm{Hp}$ genotype, Hp levels and IL-17 levels with coronary artery stenosis. In our study the frequencies of Hp genotypes, frequencies of Hp Alleles, Hp levels and IL-17 levels were significantly different between CVD group and control group, and among the three subgroups. It has been observed that $\mathrm{Hp}$ genotype and increased expression of Hp correlates with the Gensini score and IL-17. Importantly, we found that in group C Hp2-2 carriers have a 2.319-fold elevated risk for coronary diseases. These results indicate that $\mathrm{Hp}$ genotype is associated with extent of coronary artery stenosis, which may be a potential predictor of CAD. We also confirmed previous findings that IL-17 is a risk factor for CAD, oxidative stress and inflammation in premature coronary artery disease.

Coronary Artery Disease (CAD) is considered a complicated inflammatory disease. Immunological factors could play important roles in the vascular inflammation and the development and progression of atherosclerosis. Haptoglobin (Hp) is a $54 \mathrm{kDa}$ protein, found abundantly in the serum [4]. $\mathrm{HP}$ acts as a potent immunosuppressor of lymphocyte function and modulates the helper T-cell type 1 and type 2 (Th1/Th2) balance within the body. It has been reported that $\mathrm{Hp}$ has immunomodulatory properties, which protect against the toxic effects of free hemoglobin and iron-driven oxidative stress. Hp level has been consistently associated with inflammatorylinked infectious [5] and non-communicable diseases [6]. Higher circulating $\mathrm{Hp}$ has also been suggested to be associated 
with the risk of unstable carotid stenosis [7], high blood pressure, and elevated glucose [8], stroke and heart failure [9]. Our results show that the Hp levels in patients with CAD was significant higher than the controls. The significant value of $\mathrm{Hp}$ was associated with the Hp genotype. This means the different alleles lead to different levels of circulating $\mathrm{Hp}$ protein, which are in agreement with a previous study, that $H p$ gene was a strong genetic predictor of circulating Hp levels [10]. This could possibly explain the association of the Hp polymorphism with CAD. Hp is present in the serum of all mammals, but polymorphism is found only in humans. In humans, the $H p$ gene exists in two major co-dominant alleles, $\mathrm{Hp} 1$ and $\mathrm{Hp} 2$, in chromosome 16q22.2. The Hp alleles give rise to three major genotypes, Hp1-1, Hp2-1, and Hp2-2. Hp1 contain five exons. Hp2 contain seven exons, which likely arose from a duplication event involving exons 3 and 4, producing a $61 \mathrm{kDa}$ protein. The size of the Hp1 mRNA is $1284 \mathrm{bp}$, in contrast, Hp2 has a 177 bp longer mRNA. This variation is called "Hp common polymorphism" or "Hp copy-number variant". The highest frequency of the Hp1 allele is found in African and South American populations, and the lowest frequency is seen among Asian populations, particularly in India. To date, no other polymorphism in the $H p$ gene has been associated with cardiovascular or cerebrovascular morbidity. Hp functional properties are genotype-dependent. Hp1-1 is thought to confer protection from angiopathies; however, Hp2-2 is believed to be a major risk factor for several oxidative stress-related diseases. Hp1-1 is a better antioxidant and binds more strongly with free hemoglobin than Hp2-2 [11]. Thus, the protection conferred by the Hp 1-1 genotype against CAD development is thought to be mediated by the greater antioxidative and anti-inflammatory capacity of the Hp 1 allele [12]. The present study showed that the individuals with the $\mathrm{Hp} 2$ allele were more likely to have CVD (OR=0.630; 95\% CI 0.449-0.884, $\mathrm{P}<0.05$ ) and were found to be associated with a greater severity of coronary atherosclerosis (Hp vs. Gensini score, $\mathrm{r}=0.296, \mathrm{P}<0.05$ ), which might indicate that $\mathrm{Hp} 2-2$ protects less against oxidative stress in CVD. Thus, a low level of $\mathrm{Hp}$ or the presence of $\mathrm{Hp} \mathrm{2-2}$ phenotypes might be considered as a predictor of susceptibility to $\mathrm{CAD}$ and patient prognosis. This is in line with previous reports $[13,14]$ that acute phase Hp level might reflect the severity of oxidative stress during inflammation process, and a significant association was present between phenotypes resulting from a genotype containing a $\mathrm{Hp} 2$ allele and a higher degree of atherosclerosis in patients with acute coronary syndrome.

In view of the immunomodulatory role of $\mathrm{Hp}$ in immune response, it is of interest to investigate the relationship between $\mathrm{Hp}$ and pro-inflammatory cytokine. In the present study, a strong correlation between Hp levels and Gensini score and IL-17 were observed. And there were significant elevation on Hp levels, IL-17 levels, CRP levels and D-dimers levels in patients comparison with controls. These findings indirectly demonstrated that $\mathrm{Hp}$ were related to the complexity and severity of atherosclerosis and suggested the pro-atherogenic role in the development of atherosclerosis. Commenting on IL-17, our data are in favor of an indirect pro-atherosclerotic role of IL-17, in agreement with data from Tarantino's studies [15]. IL-17 is a crucial proinflammatory cytokine, belonging to Th17 type T-cell group, which is one of the major T-cell phenotype responsible from autoimmune response. As a major source of IL-17, Th17 cells have been primarily used for assessing the role of IL-17 in atherosclerosis development [16]. Some studies have shown that Th17 cells play an important role in the onset of Acute Myocardial Infarction (AMI) [17-19]. Accordingly, IL-17 may be useful as a predictor of CAD incidence. The main function of IL-17 appears to be the regulation of local tissue inflammation via the coordinated expression of pro-inflammatory cytokines and chemokines [15]. It could induces apoptosis of vascular endothelial cells by activating caspase- 3 and caspase- 9 and by increasing the Bax/ Bcl-2 ratio [20], and enhances T-cell priming and stimulates fibroblasts, endothelial cells, macrophages, epithelial cells to produce multiple proinflammatory cytokines such as IL-1, IL-6, IL-8, IFN- $\gamma$, and thereby potentially enhance plaque instability by promoting a pro-inflammatory and proatherogenic phenotype [21,22]. In our study IL-17 levels were significantly different between CVD group and control group, and among the three subgroups. This was consistent with previous reports that serum concentrations of IL-17 mirror the impaired coronary circulation [23]. Moreover, of relevance to atherosclerosis, IL-17 has been shown to promote atherosclerotic progression by several pathways including induces apoptosis of vascular endothelial cells and regulates inflammatory processes. IL-17 may be a highly important cytokine in the pathogenesis of CVD. Hp may have a role in reducing the severity of inflammation and modulates IL-17 production. In this study, IL-17 correlated with Gensini score and HP levels, which supports the idea of a possible role of HP in reducing the severity of IL-17-mediated inflammatory processes. However, it seems that the response of IL-17 is not always linked to the response of Hp genotype. Other studies have also shown controversial results about the involvement of Th17cells and IL17 in atherogenesis process [24]. Thus, further studies are clearly needed to elucidate the net effect of IL-17 levels in atherosclerotic disorders and how HP gene polymorphisms affect disease pathogenesis.

\section{Acknowledgments}

The research team acknowledges the support of the Medical Research Foundation from Department of Health of Shandong, Shandong, PR China.

\section{References}

1. Gensini GG. A more meaningful scoring system for determining the severity of coronary heart disease. Am J Cardiol 1983; 51: 606.

2. Vijetha S, Veena M, Krishnananda P. Correlation of serum homocysteine levels with the severity of coronary artery disease. Ind J Clin Biochem 2014; 29: 339-344.

3. Koch W, Latz W, Eichinger M. Genotyping of the common haptoglobin HP 1/2 polymorphism based on PCR. Clin Chem 2002; 48: 1377-1382. 
4. Levy AP, Asleh R, Blum S. Haptoglobin: basic and clinical aspects. Antioxid Redox Signal 2010; 12: 293-304.

5. Kasvosve I, Speeckaert MM, Speeckaert R. Haptoglobin polymorphism and infection. Adv Clin Chem 2010; 50: 23-46.

6. Asleh R, Marsh S, Shilkrut M. Genetically determined heterogeneity in hemoglobin scavenging and susceptibility to diabetic cardiovascular disease. Circ Res 2003; 92: 1193-1200.

7. Ijas P, Saksi J, Soinne L. haptoglobin 2 allele associates with unstable carotid plaque and major cardiovascular events. Atherosclerosis 2013; 230: 228-234.

8. Hamalainen P, Saltevo J, Kautiainen H. Erythropoietin, ferritin, haptoglobin, hemoglobin and transferring receptor in metabolic syndrome: a case control study. Cardiovasc Diabetol 2012; 11: 116.

9. Holme I, Aastveit AH, Hammar N. Inflammatory markers, lipoprotein components and risk of major cardiovascular events in 65,005 men and women in the Apolipoprotein MOrtality RISk study (AMORIS). Atherosclerosis 2010; 213: 299-305.

10. Froguel P, Ndiaye NC, Bonnefond A. A genome-wide association study identifies rs2000999 as a strong genetic determinant of circulating haptoglobin levels. PLoS One 2012; 7: 32327.

11. Van Vlierberghe H, Langlois M, Delanghe J. Haptoglobin polymorphisms and iron homeostasis in health and in disease. Clin Chim Acta 2004, 345: 35-42.

12. Costacou T, Levy AP. Haptoglobin genotype and its role in diabetic cardiovascular disease. J Cardiovasc Transl Res 2012; 5: 423-435.

13. Chiang KH, Kao YT, Leu HB. Higher post- acute myocardial infarction plasma haptoglobin level is associated with poor long-term overall survival. Int J Cardiol 2017; 229: 102-107.

14. Buljubasic N, Oemrawsingh RM, Smeets MB. Haptoglobin polymorphism in relation to coronary plaque characteristics on radiofrequency intravascular ultrasound and nearinfrared spectroscopy in patients with coronary artery disease. Int J Cardiol 2016; 221: 682-687.

15. Tarantino G, Costantini S, Finelli C. Is serum Interleukin-17 associated with early atherosclerosis in obese patients? J Transl Med 2014; 12: 214.
16. van Bruggen N, Ouyang W. Th17 cells at the crossroads of autoimmunity, inflammation, and atherosclerosis. Immunity 2014; 40: 10-12.

17. Zhao Z, Wu Y, Cheng M. Activation of Th17/Th1 and Th1, but not Th17, is associated with the acute cardiac event in patients with acute coronary syndrome. Atherosclerosis 2011; 217: 518-524.

18. Zeng Z, Yu K, Chen L. Interleukin-2/Anti-Interleukin-2 Immune Complex Attenuates Cardiac Remodeling after Myocardial Infarction through Expansion of Regulatory $\mathrm{T}$ Cells. J Immunol Res. 2016, 2016: 8493767

19. Akhavanpoor M, Akhavanpoor H, Gleissner CA. The two faces of Interleukin-17A in atherosclerosis Interleukin-17A and atherosclerosis. Curr Drug Targets 2016.

20. Zhu F, Wang Q, Guo C. IL-17 induces apoptosis of vascular endothelial cells-a potential mechanism for human acute coronary syndrome. Clin Immunol 2011; 141: 152-160.

21. Ng HP, Burris RL, Nagarajan S. attenuated atherosclerotic lesions in apoe-fcgamma-chain-deficient hyperlipidemic mouse model is associated with inhibition of th17 cells and promotion of regulatory $\mathrm{t}$ cells. J Immunol 2011; 187: 6082-6093.

22. Griffin GK, Newton G, Tarrio ML. IL-17 and tnf-alpha sustain neutrophil recruitment during inflammation through synergistic effects on endothelial activation. J Immunol 2012; 188: 6287-6299.

23. Simon T, Taleb S, Danchin N. Circulating levels of interleukin-17 and cardiovascular outcomes in patients with acute myocardial infarction. Eur Heart J 2013; 34: 570-577.

24. De Boer OJ, Van der Meer JJ, Teeling P. Differential expression of interleukin-17 family cytokines in intact and complicated atherosclerosis. J Pathol 2010; 220: 499-508.

\section{*Correspondence to}

Xiaoying Ma

Department of Medicine

Qingdao Municipal Hospital

Qingdao University Medical College

PR China 